\title{
ATUAÇÃO DO PSICÓLOGO NOS HOSPITAIS DA GRANDE VITÓRIA/ES: UMA DESCRIÇÃO ${ }^{1}$
}

Luziane Zacche Avellar

\begin{abstract}
RESUMO. O presente trabalho teve como objetivo verificar a existência de profissionais de psicologia nos hospitais da Região Metropolitana da Grande Vitória/ES, descrever as atividades por eles desenvolvidas e seus objetivos de trabalho, e discutir a inserção destes profissionais nos hospitais. Foram entrevistados 23 psicólogos que atuavam em hospitais no ano de 2006. Os resultados revelaram que a distribuição de profissionais na região é irregular, com maior concentração na capital. Ainda predomina o modelo tradicional de atendimento, baseado na assistência clínica individual. Apesar disto, alguns profissionais relatam experiências profissionais que revelam a busca por novas formas de atuação no território hospitalar e propõem novas ações, com preocupação voltada para a atenção integral à saúde.
\end{abstract}

Palavras-chave: Saúde; psicologia da saúde; hospital; psicologia hospitalar.

\section{PRACTICE OF PSYCHOLOGIST IN HOSPITALS IN GRANDE VITÓRIA/ES: A DESCRIPTION}

\begin{abstract}
This study aimed to verify the existence of psychologists in hospitals in the Metropolitan Region of Grande Vitoria/ES, to describe the activities undertaken by them, their work goals and discuss the integration of these professionals in hospitals. We interviewed 23 psychologists who worked in hospitals in 2006. The results revealed that the distribution of professionals in the region is uneven, there is a greater concentration in the capital. There is still a predominance of a traditional model of care, focusing predominantly on a individual clinical model. Despite this, some practitioners professionals report experiences which reveal a search for new ways of working within the hospital and offer new shares with a focused concern for comprehensive health care.
\end{abstract}

Key words: Health; health care psychology; hospital; hospital psychology.

\section{PAPEL DEL PSICÓLOGO EN LOS HOSPITALES EN VITÓRIA/ES: UNA DESCRIPCIÓN}

RESUMEN. Este estudio tuvo como objetivo verificar la existencia de la psicología profesional en los hospitales de la Región Metropolitana de Vitória, describir las actividades que desarrollaron sus objetivos de trabajo y discutir la integración de estos profesionales en los hospitales. Hemos entrevistado a 23 psicólogos que trabajaban en hospitales en 2006. Los resultados mostraron que la distribución de los profesionales en la región es desigual, hay una mayor concentración en la capital. Todavía hay un predominio de un modelo tradicional de atención, centrándose principalmente en un individuo modelo clínico. A pesar de esto, algunos participantes relacionados con la experiencia laboral que muestra una búsqueda de nuevas formas de actuar dentro del hospital y proponer nuevas acciones en el interés se centró en la atención integral de salud.

Palabras-clave: Salud; psicología de la salud; hospital; psicología hospitalaria.

Nos últimos anos tem-se observado crescente inserção de profissionais de psicologia nos serviços hospitalares (Yamamoto, Cãmara, Silva, \& Dantas, 2001; Costa, Barbosa, Francisco, Estanislau, Wanderley; Bastos, \& Morais, 2009; Tonetto \&
Gomes, 2007; Vieira, 2010). Tal mudança tem sido sustentada pela sempre maior aceitação do modelo biopsicossocial em saúde.

A ampliação do conceito provocou importantes transformações na área. Spink, (2009) aponta para a

Apoio: FAPES - Fundação de Amparo a Pesquisa do Espírito Santo, Edital n. 01/2005.

Doutorado em Psicologia (Psicologia Clínica) pela Pontifícia Universidade Católica de São Paulo, Brasil(2002). Professora do Programa de PósGraduação em Psicologia da Universidade Federal do Espírito Santo, Brasil. 
ressignificação da causalidade na explicação da doença, que passa a ser vista como um fenômeno complexo, precisando ser abordada de forma a integrar as dimensões biológica, psíquica e social.

Nigro (2004) entende que a mudança de paradigma na área da saúde colocou em cena novos atores, novos espaços e uma nova linguagem. Desta maneira, a Psicologia entrou no hospital trazendo um olhar que abre espaço para uma escuta diferenciada, na qual a história do paciente internado se torna significativa para entender a história da sua doença. Os aspectos psicossociais também passam a ser enfatizados quando se tenta compreender a multicausalidade das doenças psíquicas. Tal postura dificulta o entendimento do hospital ou qualquer estabelecimento de saúde pelo viés do domínio da medicina e do saber médico, que privilegia o corpo biológico.

Para Pusch (2010), a construção de uma nova práxis no espaço interdisciplinar no ambiente hospitalar deve ser norteada por princípios éticos e humanitários que levem em consideração a necessidade de relações de colaboração entre os profissionais de saúde, o paciente e seus familiares. Bonfim (2009), ao abordar a questão da humanização em centros de terapia intensiva, também frisa a importância de manter o foco nas necessidades dos pacientes, familiares e profissionais. Ao eleger a CTI como espaço para seu estudo, enfatiza a necessidade de os atores sociais envolvidos considerarem a questão da possibilidade da morte.

A medicina moderna, em especial a medicina hospitalar que tem por objeto os casos agudos e de emergência, acaba por deixar em segundo plano o sofrimento psíquico decorrente da doença e da possibilidade da morte e muitas vezes não abre um espaço de escuta para a ruptura provocada no momento anterior e no posterior à doença, nem para os danos psicológicos provocados pelo processo do adoecimento.

A experiência de hospitalização, para Orfali (citado por Spink, 2009) implica uma construção em três planos. $\mathrm{O}$ primeiro deles refere-se à relação do paciente com sua doença e, neste contexto, a necessidade de este ser tratado como doente - frágil e inseguro diante da experiência da dor e da possibilidade de morte. Outro plano é o da situação de emergência, em que se exige uma assistência médica de excelência e, passada a urgência, surge à relação do paciente com o sistema de cura. Nesse nível o paciente quer ser tratado como pessoa e a estratégia utilizada para isto é a relacional. O terceiro plano é o da relação do paciente com a organização hospitalar e com o próprio sistema de saúde e a exigência de esse paciente ser respeitado na sua condição de usuário de serviços públicos de saúde.

Tais aspectos relacionados aos processos de adoecimento incidiram diretamente na ampliação de oferta de trabalho para o psicólogo. Neste sentido, vale mencionar dois fatos importantes: a fundação da Sociedade Brasileira de Psicologia Hospitalar (SBPH), ocorrida em 1997, e o reconhecimento da Psicologia Hospitalar como especialidade por parte do Conselho Federal de Psicologia, ocorrido em 2002. Esses fatos contribuíram para a ampliação da produção do conhecimento científico e para a promoção e reconhecimento do trabalho do profissional que se dedica a esta área. A partir disto, o hospital passou a representar um novo espaço de interesse, inserção e sedimentação do profissional de psicologia, o que exigiu desse profissional uma revisão teórico-prática e das instituições formadoras uma reforma da formação acadêmica do psicólogo (Yamamoto \& Cunha,1998; More, Crepaldi, Gonçalves \& Menezes, 2009).

Um levantamento nacional encomendado pelo Conselho Federal de Psicologia ao IBOPE no ano de 2004 mostrou que 55\% dos psicólogos tinham como principal atividade o atendimento clínico individual ou em grupo e que $45 \%$ dos profissionais entrevistados realizavam esse atendimento em consultório particular e apenas $4 \%$ em hospitais, o que conferia à psicologia exercida em hospitais a $6^{\text {a }}$ colocação no universo de 1.673 respondentes.

No ano de 2002 a Comissão Gestora da Sessão ES do CRP-04 desenvolveu um projeto denominado "Quem somos? Onde estamos? O que fazemos? - O perfil do psicólogo no estado do Espírito Santo", com o propósito de realizar um diagnóstico dos profissionais que atuavam naquele estado. Naquele ano o número aproximado de inscritos no CRP era de mil psicólogos, atuando em todo o estado. Foram entrevistados 788 profissionais (80\% da categoria) e verificou-se que apenas $17,9 \%$ deles declararam trabalhar na área hospitalar. A grande maioria (70,3\%) declarou trabalhar na área clínica.

Se por um lado podemos considerar como positiva a abertura de mercado para o psicólogo, por outro, temos que atentar para a impossibilidade de transposição de modelos de atuação. O referencial clínico tradicional, ainda predominante, não dá conta de atender às demandas impostas pela realidade hospitalar, fato que chama a atenção para a 
necessidade de construir formas específicas de intervenção neste novo território.

Segundo Muylaert (1995), os psicólogos não possuem uma prática específica nos hospitais. É difícil delimitar o campo de ação, devido às diferentes demandas. O trabalho do psicólogo não se restringe aos pacientes. A fragilização destes pela doença e pela dor repercute também na equipe de saúde, que, ao prestar cuidados, é sensibilizada pelas queixas e desejos dos pacientes.

Um estudo realizado em hospitais do Rio Grande do Norte (Yamamoto e Cunha, 1998) demonstrou que os locais onde atuavam os psicólogos dentro de um hospital eram bastante diversificados. Além das salas de Psicologia e das enfermarias, eles podiam também trabalhar em qualquer lugar em que fosse necessária sua presença, como o leito do paciente, as salas de espera, o pátio, os centros cirúrgicos e outros. Os autores indicaram que, se por um lado, isso poderia indicar uma grande dificuldade do psicólogo em encontrar o seu lugar no hospital, por outro, poderia significar uma maior diversificação nas áreas de atuação dos profissionais de Psicologia.

Spink (2009) aponta a necessidade de rever a formação profissional:

Ao se afastar da situação paradigmática delineada nos nossos cursos de graduação, de prática psicológica como profissional liberal centrada em consultório e privilegiando a esfera individual, se faz necessário expandir o referencial teórico da graduação de forma a compreender tanto o contexto - seja este a realidade social mais ampla, ou a realidade institucional - quanto a polimorfia das representações constituídas na e constituintes dessa realidade institucional. ( p.139)

O trabalho do psicólogo na área da saúde pública em Vitória - ES tem sido tema de constantes debates nos últimos anos. A Universidade Federal do Espírito Santo, a $16^{\mathrm{a}}$ Região do Conselho Regional de Psicologia - ES - e as instituições particulares de ensino têm tido papel importante na fomentação deste debate; entretanto podemos avaliar a atuação do psicólogo no âmbito hospitalar como ainda incipiente.

Considerando-se a atualidade do tema e a importância das atividades realizadas por psicólogos em hospitais, a presente pesquisa justifica-se pela relevância científica, ao produzir conhecimentos a respeito da atuação do psicólogo no âmbito hospitalar, e pela relevância social, no sentido de gerar conhecimentos que auxiliam no desenvolvimento e aprimoramento de intervenções na área da saúde pública na região da Grande Vitória ${ }^{2}$.

O nosso objetivo foi verificar a existência de profissionais de Psicologia nos hospitais da Região Metropolitana da Grande Vitória no ano de 2006, bem como descrever as atividades por eles desenvolvidas, seus objetivos de trabalho, a prática de trabalho em equipe multiprofissional e as principais dificuldades encontradas para a realização de seu trabalho.

\section{MÉTODO}

\section{Procedimentos de coleta de dados:}

Após aprovação do Comitê de Ética em Pesquisa iniciou-se a coleta de dados, que ocorreu no período de fevereiro a junho do ano de 2006. Inicialmente realizou-se um levantamento junto à Secretaria Estadual de Saúde do Espírito Santo para verificar se havia psicólogos atuando em hospitais gerais na Grande Vitória, tendo-se encontrado quarenta profissionais trabalhando nesses hospitais. Em seguida realizou-se contato telefônico com cada um deles para apresentar-lhes sucintamente os objetivos da pesquisa e convidá-los a dela participar. Após o aceite eram agendados o dia e o horário que melhor lhes convinham para a realização da entrevista, a qual era precedida da assinatura do Termo de Consentimento Livre e Esclarecido.

Como critério de inclusão no grupo de participantes ficou estabelecido que o psicólogo deveria estar atuando em hospital geral. Foram excluídos os profissionais que apenas utilizavam as dependências anexas ao hospital para atendimento clínico privado. Com estes parâmetros obtiveram- se vinte e três participantes.

As entrevistas foram individuais. Todas foram gravadas, por escolha dos participantes, no próprio hospital onde trabalhavam. A entrevista seguia um roteiro semiestruturado em que as questões abertas tinham o objetivo de explorar os seguintes temas: (1) Atividades desenvolvidas no hospital; (2) Objetivos do trabalho; (3) $\mathrm{O}$ trabalho em equipe multidisciplinar; e

\footnotetext{
A Região Metropolitana de Vitória, ou Grande Vitória (RMGV), é formada pelos municípios de Cariacica, Fundão, Guarapari, Serra, Viana, Vila Velha e Vitória. Foi constituída pela Lei Complementar estadual 58, de 21.02.1995, e posteriormente modificada em 1999 e 2001, quando incorporou, respectivamente, os municípios de Guarapari e Fundão. Esses sete municípios abrigam quase metade da população total do Espírito Santo (46\%) e 57\% da população urbana do Estado.
} 
(4) Dificuldades mais frequentes encontradas no trabalho.

\section{Procedimento de análise dos dados}

As entrevistas foram transcritas e analisadas pelo método qualitativo proposto por Minayo (2002). O trabalho de análise consistiu na organização do material coletado e na leitura repetida das transcrições das entrevistas para preparação da análise. As categorias temáticas foram previamente estabelecidas. Foram excluídos os elementos que não seriam utilizados, condensando-se, assim, o material em conteúdos temáticos. Foram realizados recortes nos textos das entrevistas onde se verificava a presença das categorias. Após a construção destes recortes as categorias temáticas foram analisadas e interpretadas, dando corpo aos resultados apresentados a seguir.

\section{RESULTADOS $^{3}$}

Para maior clareza na apresentação dos resultados montou-se a tabela 1, que mostra a existência e distribuição de psicólogos nos hospitais dos municípios que compõem a RMGV.

Os resultados das entrevistas com os profissionais são apresentados a seguir, organizados por município.

\section{Os psicólogos nos hospitais}

O levantamento realizado junto à Secretaria de Saúde do Estado do Espírito Santo (SESA) trouxe o resultado que será apresentado na Tabela 1.

Tabela 1 - Número de Profissionais de Psicologia nos Hospitais da Região Metropolitana da Grande Vitória no Ano de 2006

\begin{tabular}{|c|c|c|c|c|}
\hline Município & $\mathbf{N}^{0}$ de hospitais & $\mathbf{N}^{0}$ de Psicólogo nos hospitais & Profissionais entrevistados & Tipo de vínculo \\
\hline Serra & 03 & 06 & 04 & Contrato/concurso \\
\hline Vitória & 10 & 25 & 18 & Contrato/concurso/voluntariado \\
\hline Cariacica & 02 & 01 & 00 & Contratado/atuação em RH \\
\hline Viana & 01 & 00 & 00 & \\
\hline Vila Velha & 08 & 07 & 01 & Contrato/concurso \\
\hline Guarapari & 03 & 01 & 00 & Não possui vinculo com o hospital \\
\hline Fundão & 01 & 00 & 00 & \\
\hline Total & 28 & 40 & 23 & \\
\hline
\end{tabular}

Verifica-se que a distribuição de psicólogos nos hospitais da Grande Vitória era irregular na época da coleta dos dados, tendo uma maior concentração na capital. Em dois municípios os hospitais não contavam com profissionais de psicologia em seu quadro funcional (Viana e Fundão), em um município havia um psicólogo atuando apenas na área de recursos humanos (Cariacica) e em um município (Guarapari) havia apenas um psicólogo, o qual utilizava as dependências do hospital para atendimento privado.

Uma possível explicação para a maior concentração de profissionais no município de Vitória seria o fato de estes terem carga horária parcial e, deste modo, poderem desenvolver outras atividades

3 Por razões de ordem ética, não mencionamos os nomes dos hospitais nem identificamos os profissionais que participaram da pesquisa. Tal procedimento não traz prejuízos para a apresentação dos dados , uma vez que o foco da pesquisa foi a descrição do conjunto das atividades desenvolvidas pelos psicólogos que exercem suas atividades nos hospitais da GV. profissionais concomitantemente com a desenvolvida no hospital, vantagem que influenciaria na escolha do local de trabalho. Chamou a atenção o número de profissionais voluntários encontrados em um dos hospitais pesquisados, aspecto que será discutido mais adiante.

\section{As entrevistas com os profissionais}

O município da Serra possui três hospitais e, ao todo, seis psicólogos (dois em cada um dos hospitais), quatro dos quais foram entrevistados. As atividades descritas pelos profissionais entrevistados foram: atendimento clínico individual (atendimento psicoterápico, escuta analítica (sic), psicoterapia breve, focal e emergencial, trabalho recreativo); acompanhamento do paciente (entrevista ao paciente, observação diária de recém-nascidos e crianças internadas, suporte psicológico a pacientes terminais, verificação diária de entrada e saída de pacientes); acompanhamento dos familiares (entrevistas com acompanhantes/familiares e oficinas com mães 
internadas na maternidade); atendimento em grupo (grupo terapêutico, oficinas, dinâmicas, trabalho em grupo e grupo de mães); elaboração de parecer técnico (elaborar laudos psicológicos e atender a solicitações de outros profissionais para avaliação psicológica dos pacientes); e, supervisão de estagiário.

Das atividades descritas, as mais citadas foram o atendimento clínico individual, que era realizado por todos os profissionais entrevistados. Seguem-se, pela ordem do número de citações, o acompanhamento do paciento, o acompanhamento de familiares, o atendimento em grupo, a elaboração de parecer técnico e a supervisão de estágio. Cada uma das duas últimas foi citada por apenas um entrevistado.

Os profissionais entrevistados no município da Serra descreveram como objetivos de trabalho minimizar/aliviar o sofrimento provocado pela hospitalização/internação e trabalhar o suporte emocional com vista ao bem-estar do paciente e dos familiares, além de abrir um espaço para que o paciente fale sobre ele. Vejamos o fragmento de uma das entrevistas:

\section{O objetivo é que contribua para um menor sofrimento; talvez para uma adesão maior ao tratamento, porque à medida que a pessoa fala para a gente o que ela está passando, ela vai se percebendo, ela vai se vendo, ela vai reformulando muitas coisas, ressignificando muitas histórias, muitas ideias, ela vai refletindo mesmo. A mudança de comportamento é simplesmente uma consequência da escuta. (profissional entrevistado)}

Quando lhes foi perguntado se havia equipe multidisciplinar, dois afirmaram atuar em equipes multidisciplinares. Os profissionais de saúde citados foram assistentes sociais, enfermeiros e médicos; entretanto as equipes atuavam por meio de reuniões, geralmente não sistematizadas em relação ao horário e a participantes, atendendo a demandas específicas, conforme se pode observar no seguinte fragmento de entrevista:

Ainda não existem também [trabalhos multi ou interdisciplinares]. Existem esses momentos que eu pergunto aos médicos "Como está fulano? Como é que está o prognóstico dele?”; eu que faço este link; mas não existe, por exemplo, como já participei em outra instituição: um dia, tal horário, junta todo mundo e discute ali. (profissional entrevistado)
As dificuldades relatadas por estes profissionais foram: a inserção e atuação do psicólogo no contexto hospitalar, formação acadêmica insuficiente, desvalorização ou desconhecimento do trabalho do psicólogo pelos demais profissionais, falta de espaço físico para desenvolver suas atividades no hospital, solicitação para "apagar incêndio", falta de privacidade no atendimento no leito e o fato de trabalharem "sozinhos".

O município de Vitória conta com dez hospitais, que no período da coleta de dados tinham em seus quadros, ao todo, vinte e cinco psicólogos, dos quais dezoito foram entrevistados (sete profissionais eram voluntários). As atividades descritas pelos profissionais entrevistados foram: atendimento clínico individual (ambulatório); implantação da Política Nacional de Humanização, proposta recente do Sistema Único de Saúde - SUS; grupos de discussão sobre saúde no trabalho (oficinas); grupos gestores do processo de trabalho; pesquisas (Comunidade Ampliada de Pesquisa); e Ouvidoria (os usuários podem fazer queixas e reclamações do serviço por meio do ouvidor, o qual procurará saná-las e dar um "feedback" ao usuário; o trabalho da Ouvidoria está atrelado ao da educação permanente, pois muitas vezes o problema está no mau atendimento); participação em programas específicos: como diabetes e DST/AIDS; trabalho com alcoolistas, familiares e pessoas a eles ligados; palestras como trabalho preventivo; grupos na enfermaria; elaboração de parecer; preparação pré-cirúrgica; consultas individuais pós-operatórias; trabalho na UTI neonatal (com o bebê internado, com o casal e familiares); preparação do clima para a internação na UTI; atendimento para orientação de funcionários; grupo de orientação a pais; grupo terapêutico com adolescentes e jovens e grupo terapêutico com mulheres com mais de 50 anos; atendimento a programas interdisciplinares, como PREMA - Programa de Reabilitação da Mulher Mastectomizada - e Programa de Radioterapia; Interconsultas e supervisão de estagiários.

Os profissionais entrevistados do município de Vitória mencionaram de maneiras diversas os seus objetivos de trabalho, diversidade que entendemos dever-se aos diferentes programas dos quais faziam parte. Passamos a citar alguns destes objetivos:

O objetivo do meu trabalho aqui eu acho que é lidar com a dor, é minimizar a dor do paciente (a dor da internação, a dor da perda, da espera) ... É lidar com a dor, é você minimizar a dor do paciente, do familiar e às vezes até do próprio staff, que 
fica muito angustiado, porque não sabe o que vai fazer com o paciente. (profissional entrevistado)

É pôr esse sujeito para falar, pois a medicina através de seu método científico anula a subjetividade do sujeito ... é convocar esse sujeito para falar, é criar, organizar e administrar esses lugares de escuta, para que haja espaço de fala entre as equipes. É lidar, criar uma demanda dentro do próprio hospital. (profissional entrevistado)

Eu acho que é dar este suporte psicológico durante o tratamento, que é muito necessário você ter esse olhar integral, como eu falei, pra essa pessoa que chega, pra história dela, pra família dela, não um olhar só pra doença. (profissional entrevistado)

Com relação ao trabalho em equipe multidisciplinar, quatro profissionais avaliaram positivamente a sua participação nas equipes. Os demais profissionais afirmaram que existia o trabalho em equipe, mas o descreveram como pouco sistematizado, como se pode observar neste fragmento de uma das entrevistas:

\begin{abstract}
Aqui não existe trabalho multi ou interdisciplinar. $O$ que existe são encontros informais com os outros profissionais, mais frequentemente com a assistente social, que pede ajuda em alguns casos. Geralmente a gente fala rapidinho no corredor mesmo.
\end{abstract}

As principais dificuldades mencionadas pelos profissionais nos hospitais do município de Vitória foram: a questão da hierarquia, o fato de $o$ psicólogo ser chamado para "apagar incêndio"(sic), dificuldades inerentes ao discurso da medicina, falta de espaço físico adequado para os atendimentos, a grande demanda pelo trabalho do psicólogo em contraposição ao pequeno número de profissionais atuando, dificuldade em falar com a equipe médica, o desconhecimento, por parte dos outros profissionais, do trabalho do psicólogo no hospital.

O município de Vila Velha contava com oito hospitais, nos quais atuavam sete psicólogos. Quatro deles prestavam atendimento voltado à comunidade, utilizando um espaço anexo ao hospital, por isso não estavam envolvidos em sua dinâmica, ou seja, apesar de utilizarem suas dependências físicas, não participavam das rotinas hospitalares e assim não atenderam aos critérios de inclusão no grupo de participantes. Apenas um profissional concordou de participar da pesquisa. Este descreveu como principal atividade o atendimento de crianças internadas e de seus familiares. Com os pais ou acompanhantes realizavam trabalho em grupo, num espaço de discussão aberto na própria enfermaria. Esses encontros coletivos não eram programados, aconteciam na medida em que os assuntos iam surgindo entre os acompanhantes. O objetivo de trabalho descrito pelo profissional foi "abordar temáticas que se relacionavam com o cotidiano daquelas pessoas e que de alguma maneira pudesse ajudá-las na promoção de saúde."

Segundo o profissional entrevistado, não existia trabalho em equipe multiprofissional. As dificuldades mencionadas foram: falta de um espaço mais adequado para os atendimentos de Psicologia, o não reconhecimento do trabalho do psicólogo no âmbito hospitalar e o desconhecimento das funções do psicólogo no contexto hospitalar.

\section{DISCUSSÃO}

Nesta pesquisa partimos do objetivo de verificar se havia psicólogos atuando nos hospitais da RMGV e descrever as atividades por eles realizadas, oferecendo uma contribuição no sentido de gerar conhecimentos que pudessem auxiliar no desenvolvimento e aprimoramento de intervenções na área da saúde, especialmente em hospitais da região.

Nossa primeira constatação foi que a participação do profissional de psicologia nas instituições hospitalares na GV ainda era tímida no ano em que realizamos a coleta dos dados. É fato que a inserção profissional num determinado campo não se dá em um curto espaço de tempo. Alguns de nossos colaboradores relataram que uma das dificuldades para a inserção do psicólogo no hospital era o desconhecimento, por parte de outros profissionais, do papel do psicólogo no âmbito hospitalar.

Vieira (2006) apresenta um dado semelhante: em seu grupo de entrevistados praticamente todos os profissionais apontaram a necessidade de um período de adaptação da equipe de saúde à presença do psicólogo, de um período de construção da relação profissional. A autora acredita ainda que a dificuldade mencionada pode ser consequência do fato de a psicologia ter sido uma das últimas profissões a entrar no hospital e de nem todos os 
profissionais de saúde ainda terem claro qual o papel a ser desempenhado pelo psicólogo nessa instituição.

Apesar das dificuldades mencionadas por nossos participantes, as quais referidas também por Vieira (2006); Tonetto e Gomes (2007) afirmam que há um crescente interesse pelo trabalho psicológico em hospitais, e que a Psicologia, como ciência, está preparada para dar sua contribuição de modo eficiente e seguro.

Outro ponto que merece destaque refere-se à formação acadêmica. Todos os entrevistados julgavam ter tido uma formação deficiente. Tal dado vai ao encontro do que Yamamoto, Trindade e Oliveira (2002) apontam em seu trabalho ao afirmarem que a formação universitária é insuficiente e sempre exige uma complementação sob as formas de pós-graduação e supervisão.

Outro ponto diz respeito à desvalorização do profissional que é sentida nas relações dentro da equipe, marcadas por forte hierarquia, e à supervalorização do profissional de medicina. Além disso, a falta de reconhecimento se expressa também na ausência de contratação do profissional, mesmo se reconhecendo sua necessidade. Todos os entrevistados mencionaram o grande volume de trabalho para ser executado por poucos profissionais. More, Crepaldi, Gonçalves e Menezes (2009), ao fazerem um balanço das conquistas, contradições e avanços da área, chamam a atenção para o risco de o psicólogo se tornar um mero executante das queixas e ordens dos profissionais que fazem o encaminhamento ao serviço de psicologia. Tal situação sustentaria ações fragmentadas e preconceitos relacionados ao paciente, à equipe de saúde e ao contexto.

Vale destacar o alto índice de profissionais voluntários presentes em um mesmo hospital no município de Vitória, fato que demonstra a fragilidade da vinculação, expressa nas poucas horas de trabalho dedicadas à instituição associadas às necessidades que o profissional tem de receber salário pelo seu trabalho. Moniz e Araújo (2008), problematizando a questão do voluntariado no contexto hospitalar, chamam atenção para a necessidade de reflexão sobre as consequências da resposta do voluntariado às insuficiências do Estado no atendimento às demandas na área da saúde. Reconhecem como importante a emergência do chamado terceiro setor, mas entendem que o trabalho voluntário não deve ser valorizado apenas em razão dos interesses econômicos associados. Compartilhamos da mesma preocupação dos autores citados no que diz respeito aos resultados encontrados na presente pesquisa, pois constata-se uma incoerência: há o reconhecimento da importância do profissional de Psicologia no hospital, mas esse reconhecimento não é suficiente para sua contratação e inclusão no quadro efetivo de profissionais.

No tocante às atividades desenvolvidas pelos profissionais, grande parte dos entrevistados elegeu o atendimento clínico individual como a principal forma de atuar. Tem-se que tal modelo está alinhado a um modo de atuação chamado de "tradicional". A este respeito citaremos Yamamoto, Trindade e Oliveira (2002):

O ponto a ser colocado, aqui, não diz
respeito à novidade das práticas, mas à sua
adequação. Considerando-se que os
hospitais sejam locais privilegiados para a
modalidade de atenção terciária, uma
intervenção mais direta utilizando recursos
tradicionalmente desenvolvidos e
empregados pelos psicólogos clínicos
talvez seja a única escolha possível. Isso,
entretanto, não isenta os profissionais que
trabalham nos hospitais de demonstrar que
tais práticas resistem às críticas que têm
acompanhado o trabalho clínico
tradicional. Dito de outra forma, essa
"nova" modalidade terá herdado não
somente os recursos como também as
objeções a tal abordagem individualista.
(p. 20)

Seidl e Costa Júnior (citados por Vieira, 2006) dividem em dois os modelos de atuação. Um deles é o "modelo clínico", em que o psicólogo delimita o espaço físico para a sua atuação, geralmente ocorrendo em sala reservada para o serviço de Psicologia, com pouca interação com a equipe. Nesse modelo o foco da atuação é exclusivamente o paciente e há uma ênfase na intervenção psicoterápica, individual ou em grupo. $\mathrm{O}$ outro modelo é o "biopsicossocial " ou de " atenção integral à saúde", com atuação em diversos espaços: o psicólogo estaria "presente onde a atuação fosse necessária". Nesse modelo a equipe trabalha em conjunto; as intervenções ocorrem tanto com o paciente quanto com a família e a instituição/comunidade, e as ações são bastante diversificadas: aconselhamento, orientação, preparação pré e pós-cirúrgica, atividades educativas, intervenção breve, visitas domiciliares e outras. 
Ao fazer a transposição desta análise para os dados, observa-se que $64 \%$ dos entrevistados elegeram como forma de ação o atendimento clínico individual, enquanto $36 \%$ elegeram o atendimento em grupo. Constata-se, assim, que ainda predomina o modelo clínico na atuação profissional do grupo de entrevistados. Por outro lado, vale destacar que as outras atividades mencionadas que tiveram uma ocorrência menor estariam de acordo com o modelo chamado pelos autores de "biopsicossocial". Embora com menor frequência, as atividades que caracterizariam tal modelo foram citadas por diferentes profissionais. Isto pode indicar uma mudança na forma de atuação sinalizando uma transição do modelo clínico para o modelo biopsicossocial.

$\mathrm{O}$ que se pode observar no caso da $\mathrm{GV}$ é que, apesar de predominarem atividades que caracterizariam a atividade clínica tradicional, os profissionais estão explorando este novo território, o hospital, e propondo novas ações com preocupações voltadas a uma atenção à saúde integral e mais próxima dos ideais de humanização da assistência em saúde.

\section{CONSIDERAÇÕES FINAIS}

A pesquisa teve como objetivos verificar a existência de psicólogos nos hospitais da RMGV e descrever suas atividades. Os resultados encontrados demonstram que há contradições, avanços e retrocessos na atuação do profissional de Psicologia no âmbito hospitalar.

Questões antigas, como a rígida hierarquia no interior de algumas equipes de saúde - nas quais a figura central é o médico -, deficiências na formação profissional e a tentativa de transposição do modelo clínico tradicional para o contexto hospitalar ainda estavam muito presentes no momento da realização da pesquisa.

Além dos aspectos mencionados, é necessário reconhecer que os psicólogos entrevistados, em sua maioria, trabalhavam no sentido de aperfeiçoar sua prática profissional, sempre na busca de um modelo de atuação pautado pela ética no cuidado.

Para concluir, vale destacar que as mudanças produzidas pela substituição de um modelo exclusivamente biológico por outro que integre as mais diferentes dimensões dos processos de saúde e doença não acontecem de forma igual em todos os contextos. Daí a importância da produção e publicação de trabalhos que problematizem as diferentes situações, para que se possam acompanhar os avanços, retrocessos e contradições da área. Entende-se que toda mudança requer tempo e depende de cada região, de cada instituição e de cada equipe de trabalho.

\section{Agradecimentos}

Agradecemos à equipe executora: Ana Paula Oliveira Martins, Daiane Casagrande Lorencine, Inglid Aparecida Magri, Liana Souza Santos e Roberta Alvarenga de Almeida.

\section{REFERÊNCIAS}

Avellar, Luziane Zacche. (2006). A psicologia hospitalar na Grande Vitória: uma descrição. (Relatório técnico de pesquisa, Edital FAPES n. 01/2005).

Bomfim, R. A. (2009). Humanização no cuidado de tratamento intensivo: revisão. Revista de Administração em Saúde, 11(44), 133-142.

Conselho Federal de Psicologia (2004). Pesquisa revela perfil do psicólogo. Jornal de Psicologia CRP-SP, 141, 10-11.

Conselho Regional de Psicologia $4^{\mathrm{a}}$ Região. Comissão Gestora da Sessão ES. (2002). Quem somos? Onde estamos? O que fazemos? - O perfil do psicólogo no estado do Espírito Santo.

Costa, V. A. S. F., Barbosa, L. N. F., Francisco, A. L., Estanislau, A. C. A. L., Wanderley, E. M. T., Bastos, M. A., \& Morais, R. M. B. (2009). Cartografia de uma ação em saúde: O papel do psicólogo hospitalar. Revista da Sociedade Brasileira de Psicologia Hospitalar, 12(1), 113-134. Recuperado em 12 janeiro, de 2009 de

http://pepsic.bvsalud.org/scielo.php?script=sci_arttext\&pid=S151 6-08582009000100009\&lng=pt\&nrm=iso

Minayo, M. C. de S. (2002). Pesquisa Social: teoria, método e criatividade. Petrópolis: Vozes.

Moniz, A. L. F., \& Araújo, T. C. C. (2008). Voluntariado hospitalar: um estudo sobre a percepção dos profissionais de saúde. Estudos de Psicologia, 13(2), 149-156.

More, C. L. O. O., Crepaldi, M. A., Gonçalves, J. R., \& Menezes, M. (2009). Contribuições do pensamento sistêmico à prática do psicólogo no contexto hospitalar. Psicologia em Estudo Maringá, 14(3), 465-473.

Muylaerte, M. A. (1995). Corpoafecto: o psicólogo no hospital geral. São Paulo: Editora Escuta.

Nigro, M. (2004). Hospitalização: o impacto na criança, no adolescente e no psicólogo hospitalar. São Paulo: Casa do Psicólogo.

Pusch, R. (2010). Humanização e integralidade. Revista da Sociedade Brasileira de Psicologia Hospitalar. 13(2), 210-216. Recuperado $\begin{array}{llll}\text { em } & 12 & \text { janeiro, de } & 2009\end{array}$ http://pepsic.bvsalud.org/scielo.php?script=sci_arttext\&pid=S151 6-08582009000100009\&lng=pt\&nrm=iso

Spink, M. J. P. (2009). Psicologia Social e Saúde: práticas, saberes e sentidos. Rio de Janeiro: Vozes. 
Tonetto, A. M., \& Gomes, W. B. (2007). A prática do psicólogo hospitalar em equipe multidisciplinar. Estudos de Psicologia, 24(1), 89-98.

Vieira, C. M. A. M. (2006). A construção de um lugar para a Psicologia em hospitais de Sergipe. Dissertação de Mestrado Não Publicada, Programa de Pós Graduação em Psicologia Social, Pontifícia Universidade Católica de São Paulo, São Paulo.

Vieira, M. C. (2010). Atuação da psicologia hospitalar na medicina de urgência e emergência. Revista Brasileira de Clinica Médica, 8(6), 513-519.

Yamamoto, O. H., \& Cunha, I. M. F. F. O. (1998). O psicólogo em hospitais de Natal: uma caracterização preliminar. Psicologia Reflexão e Critica, 11(2), 345-362.
Yamamoto, O. H., Câmara, R. A., Silva, F. L., \& Dantas, C. M. B. (2001). Espaços, práticas: o que há de novo na psicologia no Rio Grande do Norte? Psicologia em Estudo, 6(2), 65-72.

Yamamoto, O. H., Trindade, L. C. B. O., \& Oliveira, I. F. (2002). O psicólogo em hospitais no Rio Grande do Norte. Psicologia USP, 13(1), 217-246.

Recebido em 01/04/2010 Aceito em 15/11/2011

\section{Endereço para correspondência:}

Luziane Zacche Avellar. Universidade Federal do Espírito Santo. Departamento de Psicologia Social e do Desenvolvimento, CEMUNI VI. Av. Fernando Ferrari, n. 514, Goiabeiras, CEP 29075-910, Vitória-ES, Brasil.E-mail: luzianeavellar@yahoo.com.br. 